\title{
Applying Social Representations to Explain Public Willingness to Use GM Foods in Taiwan: The Moderating Impact of Food Technology Neophobia-An Abstract
}

\author{
Mei-Fang Chen
}

\begin{abstract}
Recent advancements in food science and technology, from genetic engineering to nanotechnology, have raised considerable controversies and anxiety-arousing topics from social groups and the general public. The concept of social representations is "a system of values, ideas, and practices," which has great contribution to the analysis of the public understanding of various technological risks or objects. Food technology neophobia, a new psychometric tool developed by Cox and Evans (2008), can be used to explain a person's willingness to use foods produced using novel technologies. There is no basis in science for regulation specific to crops and foods improved through biotech or genetically modified organisms. Genetically modified (GM) foods have received substantial attention because they are associated with unnaturalness, moral considerations, uncertainty, and risks. This research aims to apply the concept of social representations to explain public willingness to use GM foods in Taiwan. The moderating impact of food technology neophobia was also considered. A national self-reported questionnaire survey was conducted in Taiwan in 2015. A total of 487 valid data was analyzed by moderated regression analysis. The results revealed that in terms of social representations, if the respondents adhere to technology and consider food a necessity, then they are more likely to use GM foods. However, if they demonstrate resistance to and suspicion of novelties, then they are less likely to use GM foods. When food technology neophobia was taken into account as a moderator, the negative relationship between the respondents' resistance to and suspicion of GM foods and their willingness to use GM foods was enhanced. But the relationship between the respondents treat food as a necessity and their willingness to use GM foods was changed from positive to negative. The findings can facilitate developing a clearer understanding of people's acceptance and usage of technology innovation in the food industry.
\end{abstract}

Acknowledgments This work was supported by a grant from the Ministry of Science and Technology, Republic of China (MOST 103-2410-H-036-004-MY2).

M.-F. Chen ( $\bowtie)$

Tatung University, Taipei, Taiwan, Republic of China

e-mail:mfchen@ttu.edu.tw 